\title{
Wieviel Logik braucht die Semantik? Ein Problem (nicht nur) für die wahrheitstheoretische Semantik
}

\author{
KLAUS ROBERING \\ Institut für Fachsprache, Kommunikation und Informationswissenschaft, \\ Süddänische Universität, Kolding, Dänemark
}

\begin{abstract}
The present paper is a closer examination of that version of so-called "truth-theoretic semantics" which is developed by Larson and Segal (1995). Generally, the claim of truth-functional semantics is that the semantic meta-theory of an object language is exhausted by an adequate theory of truth for this language. Here the measure of adequacy is Tarski's celebrated "criterion T", which requires for each object language sentence $A$ a sentence (of a special form) stating the truth-conditions for $A$. Larson and Segal combine the truth-theoretic view on semantics with a modular approach to linguistics. They stipulate a semantic module in which the truth-conditions for the object language sentences are derived. Since the "logic module" is, according to Larson and Segal, separated from the semantic module, these derivations can only make quite limited use of logical means. In the present paper I shall demonstrate that even in one of the most primitive sample theories which Larson and Segal develop in their monography, it is necessary to use much more of logic than they are ready to admit. Finally, I will set up and comment on a general formulation of the problem involved here, namely: how much logic is necessary in an adequate semantics.
\end{abstract}

\section{EINLEITUNG}

Die folgende Untersuchung ist einem Problem der wahrheitstheoretischen Semantik gewidmet. Die wahrheitstheoretische Semantik ist eine Spielart der formalen Semantik, die auf von Donald Davidson entwickelte Ideen zurückgeht ${ }^{1}$. Gemäß der wahrheitstheoretischen Semantik besteht die semantische Analyse einer Objektsprache $S$ im Aufbau einer formalen Theorie, in der das Wahrheitsprädikat von $S$ - also das Prädikat „... ist ein wahrer Satz der Sprache $S^{\prime \prime}$ - festgelegt wird ${ }^{2}$. Die Bedeutung eines (Aussage-) Satzes $A$ von $S$ wird durch die Wahrheitsbedingungen von $A$ festgelegt, d. h. von den Bedingungen, unter denen $A$ der Wahrheitsdefinition zufolge tatsächlich wahr ist. Die Bedeutung eines Wortes von $S$ besteht in dem Beitrag, den es zu den Wahrheitsbedingungen der $S$-Sätze liefert.

Man beachte den Unterschied zur modelltheoretischen Semantik! Entgegen dem Anschein, der in einführenden Darstellungen häufig geweckt wird, zielt die 
modelltheoretische Semantik nicht auf die Definition des Wahrheitsbegriffs einer interpretierten Objektsprache $S$, sondern definiert den Begriff des Wahrseins in einem Modell $M$ von $S$, also die Relation „... ist ein im Modell --- wahrer Satz von $S^{\prime}$. Dieser Relationsbegriff dient dann als Ausgangsbasis für die Definition weiterer semantischer Begriffe, insbesondere des Folgebegriffs. Von Modellen ist aber im Davidsonschen Ansatz gar nicht die Rede, wohl aber - natürlich - vom Wahrsein.

Larson und Segal (1995) haben eine umfassende Lehrbuchdarstellung des davidsonschen Ansatzes für natürliche Sprachen vorgelegt. Dabei zeichnet sich die von Larson und Segal (1995) vertretene Variante der wahrheitstheoretischen Semantik durch eine interessante Eigentümlichkeit vor allen anderen formalsemantischen Ansätzen aus: Während sonst in formalen Semantiken unbeschränkter Gebrauch von allen möglichen logischen Hilfsmitteln gemacht wird, sind diese beiden Autoren geradezu darum bemüht, den Gebrauch der Logik in der Semantik zu minimalisieren. Eine semantische Theorie einer Objektsprache $S$ soll diesen beiden Autoren zufolge nur so viel Logik enthalten, wie zur Erreichung des Ziele der Theorie, also zur Erfassung des Wahrheitsprädikates, notwendig ist. An ein solches Programm knüpft sich dann natürlich die Fage: Wieviel Logik braucht eigentlich die Semantik?

Ein Ziel der folgenden Ausführungen ist es, zu zeigen, dass man selbst in den einfachsten von Larson und Segal (1995) behandelten Fällen schon erheblich mehr Logik benötigt, als die beiden Autoren zuzugestehen bereit sind. Der folgende zweite Abschnitt erklärt zunächst kurz den wahrheitstheoretischen Ansatz generell und insbesondere die Version dieses Ansatzes, die Larson und Segal entwickeln. Im dritten Abschnitt wird eine sehr einfache Beispieltheorie - die zweite der von Larson und Segal (1995) behandelten näher analysiert. Es zeigt sich, dass bereits diese einfache Theorie inadäquat ist und eine Korrektur sehr viel mehr Logik in sie einbauen müsste, als ihre beiden Urheber meinen. Im vierten Abschnitt schließlich wird eine Präziserung der Titelfrage erarbeitet.

\section{Larsons und Segals wahrheitstheoretische Semantik}

\subsection{Grundgedanken des wahrheitstheoretischen Ansatzes}

Als Ziel einer Semantik für eine natürlichen Sprache gibt Davidson (1984: 55) an: „[...] give the meanings of all independently meaningful expression on the basis of an analysis of their structure". Dies erreicht man nach Davidson im Rahmen einer Wahrheitstheorie („theory of truth“) für diese Sprache, denn die Bedeutung eines Ausdrucks ist sein Beitrag zu den Wahrheitsbedingungen der Sätze, in denen er vorkommt. Eine Theorie der Wahrheit umfasst also eine Semantik. Umgekehrt kann keine Semantik als adäquat angesehen werden, "[...] unless it provides an account of the concept of truth for that language along the general lines proposed by Tarski for formalized languages"; Davidson (1984: 55). Wahrheitstheorien sind daher die Standardformen semantischer Theorien.

Der Wahrheitsbegriff wird dabei wie in Tarskis grundlegender Abhandlung von 1935 bestimmt. Tarski gibt dort eine Methode an, den Wahrheitsbegriff für unterschiedliche (interpretierte) formale Sprachen in jeweils geeigneten Metasprachen in adäquater Weise 
zu definieren, wobei Adäquatheit bedeutet, dass der Konvention W Genüge getan wird. Der hier nur interessierende erste Teil $(\alpha)$ dieser Konvention lautet in Tarskis eigener Formulierung wie folgt:

„Konvention W. Eine formal korrekte, in den Termini der Metasprache formulierte Definition des Symbols "Wr" werden wir eine zutreffende Definition der Wahrheit nennen, wenn sie folgende Folgerungen nach sich zieht:

( $\alpha$ ) Sätze, die man aus dem Ausdruck , $x \in W r$ dann und nur dann, wenn $p^{\prime \prime}$ gewinnt, indem man für das Symbol $x$ einen strukturell-deskriptiven Namen einer beliebigen Aussage der betrachteten Sprache und für das Symbol $p$ den Ausdruck, welcher die Übersetzung dieser Aussagen in die Metasprache bildet, einsetzt"; Tarski (1935: 305f.).

Ein strukturell-deskriptiver Name ist ein komplexer Term der Metasprache, der einen Ausdruck der Objektsprache durch Beschreibung seiner syntaktischen Struktur benennt. Geklammerte Strukturbeschreibungen, wie man sie üblicherweise in der Syntax verwendet, sind Beispiele solcher Namen; vgl. unten den 3. Abschnitt. Tarski verwendet solche Namen, weil eine Definition der Wahrheit rekursiv auf die syntaktische Struktur der objektsprachlichen Aussagen zurückgreifen muss. Als Argumente des Wahrheitsprädikates werden also komplexe Terme auftreten, die Variablen enthalten, auf die metasprachlicher Quantoren Zugriff haben müssen. Dies ist bei Verwendung von Anführungsnamen nicht möglich. Man kann nicht in Anführungskontexte "hineinquantifizieren“ - jedenfalls nicht in Tarskis Theorie der Anführung, wie er sie in §1 seiner Abhandlung von 1935 erklärt.

Eine Bisubjunktion (Genau-dann-wenn-Aussage), wie sie im Teil $(\alpha)$ der Konvention W beschrieben wird, nennt man auch einen „W-Satz". Gemäß der Konvention W ist also eine Definition der Wahrheit für eine Objektsprache $L$ nur dann adäquat, wenn in der Metasprache jeder W-Satz herleitbar ist. Davidson (1984: 56) verzichtet auf die Forderung, dass eine Wahrheitstheorie eine Definition des Wahrheitsbegriffs enthalten müsse, hält aber an der Forderung der Ableitbarkeit aller W-Sätze fest:

"By a theory of truth I mean a set of axioms that entail, for every sentence in the language, a statement of the conditions under which it is true. Obviously if we have a definition of a truth predicate satisfying Tarski's Convention $\mathrm{T}$ we have a theory of truth, but in general the characterization of a theory of truth demands less".

Neben der Ableitbarkeit aller W-Sätze stellt Davidson drei weitere Forderungen auf: (1.) Eine Wahrheitstheorie soll nur endlich viele Axiome enthalten; sie muss, wie man sagt, finit axiomatisierbar sein. (2.) Eine Wahrheitstheorie soll zu entscheiden gestatten, was die Bedeutung („meaning“) einer beliebig vorgelegten objektsprachlichen Aussage ist. (3.) Die metasprachlich formulierten Wahrheitsbedingungen objektsprachlicher Aussagen sollen keine Begriffe enthalten, die nicht bereits in diesen Aussagen selbst vorkommen. Insbesondere dürfen die Wahrheitsbedingungen also höchstens dann 
semantische Konzepte - wie Referenz, Wahrheit usw. - enthalten, wenn diese Begriffe selbst schon in den betroffenen objektsprachlichen Sätzen vorkommen.

\subsection{Larsens und Segals modulare Version der Wahrheitstheoretischen Semantik}

Für Larsen und Segal (1995) ist die Semantik Teil der kognitiven Linguistik, deren Gegenstand die linguistische Kompetenz muttersprachlicher Sprecher ist, die sich im impliziten sprachlichen Wissen dieser Sprecher manifestiert. Dieses implizite sprachliche Wissen, die Sprachfähigkeit („language faculty“), ist Teil des umfassenderen kognitiven Systems und ebenso wie dieses modular gegliedert. Eines seiner Module ist eben das semantische, dessen Erforschung die spezielle Aufgabe der Semantik ist. Die Konzeption dieses Moduls ist dabei ziemlich restriktiv: „In our picture, the semantic module has contents of a very specific sort. The module contains specifications of meaning for the simplest expressions of the language and rules for deducing the meanings of complex expressions on the basis of the meaning of their parts and the structural configuration in which they occur", Larson und Segal (1995: 22). Die Form des semantischen Moduls soll dabei die einer Wahrheitstheorie im Sinne Davidsons sein.

Die Restriktivität dieser Semantikkonzeption zeigt sich darin, dass Beziehungen wie Folge, Unverträglichkeit und Synonymie, die üblicherweise dem Gegenstandsbereich der Semantik zugeschrieben und etwa in der modelltheoretischen Semantik mittels des Relation des Wahrseins in einem Modell definiert werden, aus dem Rahmen dieser Disziplin hinausführen. Sie sind zwar nicht „nicht-semantisch“, aber eben „nicht-nursemantisch": sie ergeben sich vielmehr aus einer Interaktion des semantischen mit dem logischen Modul des kognitiven Bereichs. Larson und Segal (1995: 74-76) sprechen daher auch von „logicosemantic relations“. Semantisches und logisches Modul sind zwei verschiedene Module des kognitiven Bereichs, die aber selbstverständlich miteinander - und mit anderen Modulen, insbesondere dem syntaktischen - interagieren; vgl. Larson und Segal (1995: 22-24).

Nun benötigt man aber logische Schlussweisen nicht nur zur Ableitung der logischsemantischen Relationen, sondern auch schon, um aus den allgemeinen Axiomen der Wahrheitstheorie spezielle W-Sätze herleiten zu können. Allerdings sprechen Larson und Segal hier nicht von „logischen Schlussregeln“, da für sie solche Regeln ja zum Logikmodul gehören, sondern lieber von „production rules“; vgl. Larson und Segal (1995: 107). Der Name ändert aber nichts an ihrem Status als deduktive Prinzipien. Letztendlich läuft die Architketur von Larson und Segal darauf hinaus, zwei verschiedene Logiksysteme anzusetzen: ein schwaches - die "production rules“ - für das Semantikmodul und ein stärkeres für das Logikmodul. Die Logik des Semantikmoduls, die Produktionsregeln, sollen gerade einmal so stark sein, um die Adäquatheit der Wahrheitstheorie zu garantieren: Sie sollen also gestatten, W-Sätze für alle objektsprachlichen Sätze herzuleiten - und nicht mehr.

Damit sind wir bei der Frage: „Wieviel Logik benötigt eigentlich die Semantik?“ Die Antwort, die Larson auf diese Frage geben, lautet: „Relativ wenig; jedenfalls weniger als 
üblicherweise in der formalen Semantik angenommen wird und nur ein kleines Fragment der vollen klassischen Logik". Wir werden im folgenden zeigen, dass bereits eines der einfachsten Sprachfragmente, das Larson und Segal in ihrem Buch behandeln, mehr an Logik erfordert, als die Autoren annehmen: dass sie also in ihrer Wahrheitstheorie für dieses Fragment stillschweigend deduktive Prinzipien gebrauchen die sie nicht unter den Produktionsregeln aufzählen.

\section{Die Beispieltheorie PC'}

\subsection{Anschauliche Beschreibung von $\mathrm{PC}^{\prime}$}

Das Vokabular einer Wahrheitstheorie lässt sich in drei Teilvokabulare aufteilen: Zunächst hat man (1.) das syntaktische Teilvokabular, um über die Ausdrücke der Objektsprache sprechen zu können. Es enthält typischerweise Namen für die atomaren Ausdrücke der Objektsprache, Prädikate für die grammatischen Kategorien und Bezeichnungen für die syntaktischen Operationen, mit denen komplexe Ausdrücke aus einfacheren aufgebaut werden. Um allgemeine Aussagen formulieren zu können, benötigt man auch Variablen fürobjektsprachliche Ausdrücke. Ferner benötigt man (2.) semantisches Spezialvokabular, etwa das Wahrheitsprädikat. Schließlich braucht man (3.) die Ausdrücke, mit deren Hilfe man die Wahrheitsbedingungen für die objektsprachlichen Sätze formuliert, mit deren Hilfe man also den Zustand beschreibt, in dem sich die Welt befinden muss, wenn ein bestimmter objektsprachlicher Satz wahr sein soll.

Im Falle der Beispieltheorie PC' von Larson und Segal (1995: 78-88, 111f) ist die Objektsprache ein kleiner aussagenlogischer Ausschnitt des Englischen, der über eine Negation (it is not the case that) sowie über Konjunktion (and) und Adjunktion (or) verfügt. Die Sätze des Fragments werden ausgehend von drei atomaren Sätzen mit Hilfe der angeführten Junktoren gebildet. Dies wird mit Hilfe einer kontextfreien Grammatik beschrieben; s. Larson und Segal (1995: 80).

1)
(a) $\mathrm{S} \rightarrow \mathrm{S}$ ConjP,
(b) ConjP $\rightarrow$ Conj $\mathrm{S}$,
(c) $\mathrm{S} \rightarrow \mathrm{Neg} \mathrm{S}$,
(d) Neg $\rightarrow$ it is not the case that,
(e) Conj $\rightarrow$ \{and, or $\}$,
(f) $\mathrm{S} \rightarrow\{$ John ponders, Chris agrees, Jil knows Kate $\}$.

Die mit dieser Grammatik ableitbaren Ketten sind im Grunde aussagenlogische Formeln (in den Junktoren $\wedge, \vee$ und $\neg$ ) ohne disambiguierende Klammern. Sie können daher nicht in der gewöhnlichen Weise mit Hilfe einer induktiven Definition semantisch interpretiert werden, da eine solche einen eindeutigen syntaktischen Aufbau voraussetzt. Larson und Segal interpretieren daher in ihrer Theorie PC' nicht die von der Grammatik erzeugten Ketten, sondern die zugehörigen Ableitungsbäume. Diese Bäume werden in der geläufigen Weise durch Klammerterme bezeichnet, die damit zum syntaktischen Teil der Metasprache gehören. 
Larson und Segal verlangen generell von Wahrheitstheorien, dass sie „strikt lokal“ sein müssen. Das bedeutet, dass bei der semantischen Interpretation eines Baumknotens ausschliesslich auf die Interpretationen der Töchterknoten zugegriffen werden darf. Betrachten wir hierzu etwa das Beispiel

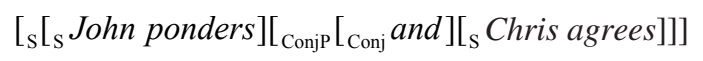

Bei der Interpretation des (einzigen) ConjP-Knoten stehen lediglich die Interpretationen des Junktors [conj and] und des Satzes [s Chris agrees] zur Verfügung. Semantisch ist der Junktor zweistellig; wir haben jedoch mit der Satzinterpretation nur ein Argument für ihn. Das wäre überhaupt kein Problem, wenn man den $\lambda$-Kalkül zur Verfügung hätten. Dann könnte man den Junktor ja einfach durch so etwas wie $\lambda x . \lambda y$. $[x \wedge y]$ interpretieren. Mit dem $\lambda$-Kalkül hätte man jedoch die Logik der Wahrheitstheorie schon ziemlich aufgebläht.

Larson und Segal haben hier nun einen recht hübschen Einfall. Sie weichen von dem strengen Buchstaben des Davidsonprogramms etwas ab, indem sie ihrer Wahrheitstheorie eine Fregesche Zutat beimengen. Statt von einem Wahrheitsprädikat gehen sie für PC' von einem Prädikatssymbol „Val“ für die zweistellige Relation zwischen semantischen Werten und den sie bezeichnenden Ausdrücken aus. Das Prädikat „Val“ gehört also zum semantischen Vokabular von PC'. Die semantischen Werte von Sätzen sind, ganz wie bei Frege, Wahrheitswerte. Zur Bezeichnung des Wahrheitswertes „Wahr“ wird der Fettbuchstabe $\mathbf{t}$ verwendet. Mit

3) $\operatorname{Val}\left(\mathbf{t},\left[{ }_{\mathrm{S}}\right.\right.$ Chris agrees $\left.]\right)$

drückt man nun also aus, dass der Satz [s Chris agrees] wahr ist. Semantische Werte haben aber nicht nur Sätze, sondern alle Ausdrücke lexikalischer Kategorien (der Grammatik (1)), also auch die Junktoren.

Und damit sind wir bei dem eigentlichen Kern von Larsons und Segals Idee. Ein zweistelliger Junktor steht in der Val-Relation zu genau denjenigen Wahrheitswertpaaren, denen durch die zum Junktor gehörige Wahrheitswertabelle $\mathbf{t}$ zugeordnet wird. Die Konjunktion hat nur einen solchen Wert: nämlich das Paar $<\mathbf{t}, \mathbf{t}>$. Demgegenüber besitzt die Adjunktion drei Werte, die den drei Wahrheitswertkombinationen entsprechen, unter denen eine Adjunktion wahr wird. Wir können diese Werte jedoch nicht notieren, da uns eine Bezeichnung für das Falsche fehlt. Es ist aber auch ohne eine solche Bezeichnung durchaus möglich, die Werte genau zu spezifizieren; vgl. das folgende (4b). Für die beiden Junktoren hat man ${ }^{3}$ :

4) (a) $\forall x y \cdot[\operatorname{Val}(\langle x, y\rangle$, and $) \leftrightarrow x=\mathbf{t} \wedge y=\mathbf{t}]$

(b) $\forall x y \cdot[\operatorname{Val}(\langle x, y\rangle$, oder $) \leftrightarrow x=\mathbf{t} \vee y=\mathbf{t}]$ 
In (4) erkennen wir auch einen weiteren Bestandteil der „logischen Maschinerie“ der Wahrheitstheorie von Larson und Segal: den Paarbildungsoperator $<>$.

Ein Objekt $x$ ist nun genau dann semantischer Wert einer Konjunktionalphrase wie [Conjp [Conj and] [s Chris agrees]], wenn es einen weiteren Wert $y$ gibt, sodass folgende zwei Bedingungen erfüllt sind: (1.) das Paar $<\mathrm{x}, \mathrm{y}>$ ist semantischer Wert der Konjunktion und (2.) $y$ ist semantischer Wert des Komplementsatzes. Im Beispiel (2) kommt man so auf:

5)

$$
\begin{aligned}
& \forall x \cdot\left[\operatorname { V a l } \left(x,\left[{ }_{\text {Conjp }}\left[{ }_{\text {Conj }} \text { and }\right]\left[\left[_{\mathrm{S}} \text { Chris agrees }\right]\right]\right) \leftrightarrow\right.\right. \\
& \left.\quad \exists y \cdot\left[\operatorname{Val}\left(\langle x, y\rangle,\left[{ }_{\text {Conj }} \text { and }\right]\right) \wedge \operatorname{Val}\left(y,\left[{ }_{\mathrm{S}} \text { Chris agrees }\right]\right)\right]\right]
\end{aligned}
$$

Man sieht, dass man hier in der Tat ohne Lambdakalkül und ohne großes mengentheoretisches Instrumentarium auskommen kann4.

Junktoren dienen dem Aufbau komplexerer Sätze aus einfacheren, d. h. schließlich aus atomaren Sätzen. Um Wahrheitsbedingungen auch für die drei atomaren Sätze der Objektsprache (s.o. (1f)) angeben zu können, muss man in der Metasprache die Zustände beschreiben können, in denen sich die Wirklichkeit befinden muss, damit die atomaren Sätze wahr sind. Hierfür nehmen Larson und Segal für die Metasprache drei Satzkonstanten an, die sich von den Namen der atomaren Sätzen der Objektsprache lediglich dadurch unterscheiden, dass sie recte gesetzt sind. Wir verwenden hier deutsche Übersetzungen und formulieren mit ihnen W-Sätze für die atomaren Sätze der Objektsprache wie etwa:

6) $\quad \operatorname{Val}(\mathbf{t}$, Phil ponders $) \leftrightarrow$ Phil überlegt.

Diese W-Sätze für die atomaren Sätze sowie die Festlegungen über die semantischen Werte der Junktoren bilden die Gruppe der „Lexical Axioms“ der Wahrheitstheorie PC'. Sie werden ergänzt durch „Phrasal Axioms“, die erklären, wie W-Sätze komplexer Ausdrücke gebildet werden. Ein Beispiel eines phrasalen Axioms ist etwa:

7) $\operatorname{Val}\left(\mathbf{t},\left[{ }_{\mathrm{S}} \mathrm{S} \operatorname{Conj} \mathrm{P}\right]\right) \leftrightarrow \exists x \cdot[\operatorname{Val}(x, \mathrm{~S}) \wedge \operatorname{Val}(x, \operatorname{ConjP})]$.

Dieses Axiom erklärt, wie man einen komplexen Satz, der aus einem Satz und einer Konjunktionalphrase aufgebaut ist, zu interpretieren hat. Es ist etwa anzuwenden, wenn man einen W-Satz für (2) herleiten will. Die Intention von Larson und Segal ist es dabei offensichtlich, dass man in (7) das zweite und dritte Vorkommnis von "S" durch den Term [s John ponders] und die beiden Vorkommnisse von „ConjP“ durch den Term [ConjP [Conj and] [s Chris agrees]] ersetzen soll.

Die nonterminalen Symbole der Grammatik (1) werden also in PC' als Variablen verwendet. Offensichtlich ist dies so gemeint, dass ein non-terminales Symbol $X$ als Variable gedeutet werden soll, die über genau die in der Grammatik erzeugbaren Baumstrukturen läuft, deren Wurzel mit diesem Symbol $X$ etikettiert ist. Also für "S“ 
soll man nur Terme für Satzbäume, für "ConjP“ nur solche für Konjunktionalphrasenbäume usw. einsetzen dürfen. Das ist für Larsons und Segals Programm ganz wesentlich, und ich werde gleich darauf zurückkommen.

Vorher muss aber noch erwähnt werden, dass die Nonterminale leider nicht immer in der gerade beschriebenen Art verwendet werden. Dem haben wir schon Rechnung getragen, als wir sagten, dass in unserem Beispiel nur das zweite und dritte Vorkommnis des S-Symbols, nicht aber das erste (als Index fungierende) zu ersetzen sei $^{5}$. Dieses Vorkommnis ist natürlich eine Konstante für ein im Baum vorkommendes Etikett; seine Ersetzung würde zu völlig unsinningen Formeln führen. In Larson und Segal Theorie PC' gibt es also mehrdeutige Symbole, die einmal als Variablen, dann aber auch als Konstanten gebraucht werden. So etwas ist in Formalisierungen, wenn auch nicht gänzlich ausgeschlossen, so doch wegen der damit verbundenen vielen Verwicklungen unerwünscht. Wir haben ja z. B. gerade gesehen, dass es Schwierigkeiten bereitet, genau zu definieren, was eine korrekte Einsetzung in ein phrasales Axiom ist. Wir werden

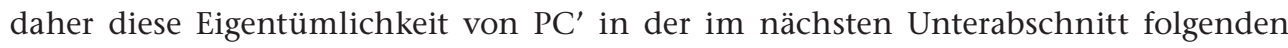
formalen Darstellung dieser Theorie eliminieren.

Für die Nonterminale bei Larson und Segal darf man, wenn überhautpt, so jeweils nur ganz spezifische Terme einsetzen. Diese Verwedungsweise dieser Symbolen zeigt, dass sie als sortenbeschränkte Variablen aufgefasst werden sollen. In sortenbeschränkten Sprachen hat man mehrere Sorten von Variablen, wobei jede Sorte einer bestimmten Art von Individuen entspricht. In $\mathrm{PC}^{\prime}$ hat man also etwa Variabeln für Satzbäume, Konkunktionalbäume, Negationsbäume und Konjunktionalphrasenbäume. Mehrsortige Theorien - also Theorien, die in mehrsortigen Sprachen verfasst sind - lassen sich aber auch als einsortige formulieren. Dazu benötigt man jedoch (neben entsprechenden Sortenprädikaten für jede einzelne Sorte) den Subjunktor $\rightarrow$, der in der Sprache von PC' gar nicht vorkommen soll. Seine Einführung (und die damit verbundene Einführung zugehöriger Schlussregeln) würde auch dem Geist des Ansatzes von Larson und Segal widersprechen. Diese wollen ja die in der Semantik verwendeten logischen Regeln möglichst minimieren. Sie verstecken dazu einen Teil der Logik der Metatheorie im Variablenapparat.

Lexikalische und phrasale Axiome alleine reichen natürlich noch nicht aus, um WSätze für alle objektsprachlichen Sätze herleiten zu können. Man muss die von diesen Axiomen gelieferten Informationen miteinander kombinieren, wozu man Schlussregeln oder - wenn man wie unsere beiden Autoren den Gebrauch logischer Terminologie in der Semantik vermeiden will - „Produktionsregeln“ („production rules“) benötigt. Betrachten wir auch hier wieder ein Beispiel. Zunächst geben wir eine neue Formulierung für (7), in der wir sortierte Variablen und Etikettenkonstanten voneinander trennen. Als Variablen für Bäume verwenden wir griechische Kleinbuchstaben. Die Sortierung wird durch Sortenindizes ausgedrückt. Hierzu benützen wir naheliegenderweise die Nonterminale der Grammatik (1), die somit sowohl als Etikettenkonstanten als auch als Sortenindizes vorkommen. Das führt aber zu keinerlei Problemen, da ja der Sortenindex 
einer Variablen semantisch kein Teilzeichen der Variable ist. Um Schreibarbeit zu sparen, notieren wir den Index einer Variable nur an deren erstem Vorkommen. Damit sieht (7) dann so aus:

8) $\forall \alpha^{\mathrm{S}} \beta^{\text {Conjp }} \cdot\left[\operatorname{Val}\left(\mathbf{t},\left[{ }_{\mathrm{S}} \alpha \beta\right]\right) \leftrightarrow \exists x^{\text {wert }} \cdot[\operatorname{Val}(x, \alpha) \wedge \operatorname{Val}(x, \beta)]\right]$.

Nun ist [s Phil ponders] ein Term der Sorte S und somit in (8) für $\alpha^{S}$ einsetzbar. Dafür benötigen wir eine „Produktion“ als Rechtfertigung: die Regel (UI) der Allbeseitigung („universal instantiation“). Wir verzichten zunächst darauf, diese genau zu formulieren ${ }^{6}$. Das Ergebnis der Regelanwendung soll aber ganz sicherlich

9) $\quad \forall \beta^{\mathrm{Conj} \mathrm{P}} \cdot\left[\mathrm{Val}\left(\mathbf{t},\left[_{\mathrm{S}}\left[_{\mathrm{S}}\right.\right.\right.\right.$ Phil ponders $\left.\left.\left.] \beta\right]\right]\right) \leftrightarrow$

$$
\left.\exists x^{\text {wert }} .\left[\operatorname{Val}\left(x,\left[{ }_{\mathrm{S}} \text { Phil ponders }\right]\right) \wedge \operatorname{Val}(x, \beta)\right]\right]
$$

sein. Eine weitere Anwendung der Allbeseitigungsregel (UI) führt dann zu:

10) $\operatorname{Val}\left(\mathbf{t},\left[_{\mathrm{S}}\left[\mathrm{S}_{\mathrm{S}}\right.\right.\right.$ Phil ponders $]\left[\left[_{\text {Conjp }}\left[{ }_{\text {Conj }}\right.\right.\right.$ and $]\left[{ }_{\mathrm{S}}\right.$ Chris agrees $\left.\left.\left.]\right]\right]\right) \leftrightarrow$

$$
\begin{aligned}
\exists x^{\text {wert }} \cdot\left[\operatorname{Val}\left(x,\left[{ }_{\mathrm{S}} \text { Phil ponders }\right]\right)\right. \\
\wedge \operatorname{Val}\left(x,\left[\left[_{\text {Conjp }}\left[{ }_{\text {Conj }} \text { and }\right]\left[\left[_{S} \text { Chris agrees }\right]\right]\right)\right] .\right.
\end{aligned}
$$

Dies hat bereits die Form eines W-Satzes, verstößt aber noch gegen die oben zum Schluss von 2.1. diskutierten Adäquatheitsbedingungen. Die rechte Seite von (10) bringt ja noch durch das dort vorkommende Val-Prädikat einen semantischen Begriff ins Spiel, der im interpretierten objektsprachlichen Ausdruck selbst gar keine Rolle spielt.

Die Strategie zur Elimination dieses Begriffs ist es, die rechte Seite von (10) so umzuformen, dass das Zeichen "Val“ nur noch in solchen Kontexten vorkommt, die in lexikalischen Axiomen wie (6) links vom Bisubjunktionszeichen stehen. Dann ersetzt man eine solche Zeichenkombination durch die entsprechende rechte Seite desselben lexikalischen Axioms. Hierzu benötigt man also eine weitere Produktion: die Ersetzungsregel für äquivalente Formel (SE) (= „substitution of equivalents“). Speziell für (10) heißt dies, dass man die rechte Seite so lange umformt, bis man

11) $\operatorname{Val}\left(\mathbf{t},\left[_{\mathrm{S}}\left[_{\mathrm{S}}\right.\right.\right.$ Phil ponders $]\left[_{\text {Conjp }}\left[_{\text {Conj }}\right.\right.$ and $]\left[_{\mathrm{S}}\right.$ Phil agrees $\left.\left.\left.]\right]\right]\right) \leftrightarrow$

$$
\operatorname{Val}\left(\mathbf{t},\left[{ }_{\mathrm{s}} \text { Phil ponders }\right]\right) \wedge \operatorname{Val}\left(\mathbf{t},\left[{ }_{\mathrm{s}} \text { Chris agrees }\right]\right)
$$

hat. Hierauf, auf das lexikalische Axiom (6) und auf ein (6) entsprechendes lexikalisches Axiom für den Satz Chris agrees, wendet man dann (SE) an, um den W-Satz 
Phil ponders $\wedge$ Chris agrees

zu erhalten.

Für den Übergang von (10) zu (12) benötigt man also die Regel (SE) sowie Umformungsregeln für die rechte Seite von (10). Letztere beseitigen Komplikationen, die durch die Auflösung der Konjunktionalphrase entstehen. Hierauf kommen wir im folgenden Abschnitt zu sprechen, in dem wir die Theorie PC' in etwas stringenterer Weise präsentieren.

\subsection{Formaler Aufbau von PC'}

PC' ist, wie wir bereits erklärt haben, eine mehrsortige prädikatenlogische Theorie erster Stufe $^{7}$. Sie verfügt zunächst über die Sorten baum, etikett und wert. Wir haben aber schon gesehen, dass diese Klassifikaiton noch nicht ausreicht. An bestimmten Stellen von PC'Formeln soll man etwa nur Bezeichnungen für Satzbäume oder nur Bezeichnungen von Konjunktionalphrasenbäume einsetzen dürfen. Wir müssen also die Sorte der Bäume noch in verschiedene Teilsorten aufspalten, wobei wir (zumindest) für jedes der nonterminalen Symbole von (1) eine entsprechende Sorte ${ }^{8}$ brauchen: also Sorten $S$, Conj, ConjP und Neg.

Ein strikter mehrsortiger Aufbau erweist sich für eine präzise Formulierung der notwendigen Regeln als zu unflexibel. Ich lege daher hier für PC' eine mehrsortige Logik zugrunde, in der wie in der Logik LE in Glubrecht/Oberschelp/Todt (1983: 74-152) die Sorten von den syntaktischen Kategorien der Logiksprache abgekoppelt sind. Die Sortenunterschiede sind nur für Individuenvariablen von Belang. Da in der Semantik keine generellen syntaktischen Gesetze formuliert werden, sind die Sorten baum und etikett damit überflüssig. In der folgenden Übersicht stellen wir die Grundausdrücke der Sprache von PC' zusammen:

- Logische Konstanten:

- Junktoren: $\neg, \wedge, \vee$ und $\leftrightarrow$

- Quantoren: $\forall$ und $\exists$

- Gleichheitszeichen: =

- Variablen

- $\quad$ der Sorte wert: $x, y, z, x_{1}, \ldots$

- $\quad$ der Sorte baums: $\rho^{s}, \sigma^{s}, \ldots$

- der Sorte baumConj: $\rho^{\text {Conj }}, \sigma^{\text {Conj }}, \ldots$

- $\quad$ der Sorte baumConjP: $\rho^{\text {ConjP }}, \sigma^{\text {ConjP }}, \ldots$

- $\quad$ der Sorte baumNeg: $\rho^{\mathrm{Neg}}, \sigma^{\mathrm{Neg}}, \ldots$ 
Klaus Robering, Wieviel Logik braucht die Semantik?

- Deskriptive Konstanten

- Aussagekonstanten: Phil überlegt, Chris stimmt zu, Jill kennt Kate;

- Individuenkonstanten: and, or, it is not the case, Chris agrees, Jill knows Kate, Phil ponders, S, Conj, ConjP, Neg, t;

- Prädikatskonstanten: die Sortenprädikatoren $\mathrm{D}^{\text {wert }}, \mathrm{D}^{s}, \mathrm{D}^{\text {baumNeg, }} \mathrm{D}^{\text {Conj }}$ und $\mathrm{D}^{\text {Conip; }}$

- Operationssymbole: [ ${ }^{1}$ und $<>$ (beide zweistellig) sowie [ $]^{2}$ (dreistellig).

Mit Hilfe der Operation [ $]^{1}$ bildet man aus einem Etikett (z. B. S) und einem Baum (z. B. Chris agrees ${ }^{9}$ ) einen neuen Baum (nämlich ${ }_{S}$ Chris agrees]), dessen Wurzel gerade das Ausgangsetikett trägt, unter dem dann der Ausgangsbaum steht. Entsprechend bildet die Operation []$^{2}$ binär verzweigende Bäume. Mit dieser Operation erhält man z. B. aus dem Etikett $S$ und den beiden Bäumen $\left[_{\mathrm{Neg}}\right.$ it is not the case] und $\left[_{\mathrm{S}}\right.$ Chris agrees] den Baum ${ }_{\mathrm{S}}\left[_{\mathrm{Neg}}\right.$ it is not the case $]\left[_{\mathrm{S}}\right.$ Chris agrees $\left.]\right]$. Aufgrund obiger Zusammenstellung bestimmen wir nun die Terme und Formeln der Theorie PC' in der üblichen Weise.

Die Begriffe des Terms und der Formel werden induktiv wie folgt bestimmt:

1. Jede Variable und jede Individuenkonstante ist ein Term.

2. Sind $e, s$ und $t$ Terme, so ${ }^{10}$ sind dies auch $\left[{ }_{\mathrm{e}} s\right]^{1}$ und $\left[{ }_{\mathrm{e}} s t\right]^{2}$ sowie $<\mathrm{s}, \mathrm{t}>$.

3. Jede Aussagenkonstante ist eine Formel.

4. Ist $t$ ein Term und $P$ eine Prädikatskonstante, so ist $P(t)$ eine Formel.

5. Sind $t$ und $t^{\prime}$ Terme, so ist $t=t^{\prime}$ eine Formel.

6. Ist $A$ eine Formel, so auch $\neg$ A. Mit $A$ und $B$ sind auch $[A \wedge B],[A \vee B]$, und $[A \leftrightarrow$ B] Formeln.

7. Ist $v$ eine Variable (irgendeiner Sorte) und $A$ eine Formel, so sind $\forall v \cdot A$ und $\exists v$. $A$ Formeln.

Wenn wir im Folgenden konkrete Formeln hinschreiben, machen wir dabei von den üblichen Klammerersparnisregeln Gebrauch. Den Sortenindex einer Variable notieren wir innerhalb einer Formel nur am ersten Vorkommnis der Variablen.

Wie üblich sagen wir, dass eine Variable $v$ in einer Formel $A$ an einer bestimmten Stelle gebunden vorkommt, wenn diese Stelle innerhalb einer Teilformel der Gestalt $Q v \cdot B(Q \in$ $\{\exists, \forall\})$ von $A$ liegt. Gibt es eine solche Teilformel nicht, so kommt $v$ an der betreffenden Stelle frei vor.

Die gerade eingeführten Begriffe des freien und des gebundenen Vorkommens einer Variable benötigt man bekanntlich zur korrekten Formulierung der Regel (UI) der Allbeseitigung (engl.: „universal instantiation“). Dies ist auch eine Regel von PC”. Larson und Segal formulieren sie (ohne einschränkende Randbedingung) wie in (13a). 
13)
(a) $\frac{\text { For any } S, F(S)}{F(\alpha)}$
(b) $\frac{\mathrm{D}^{a}(t) \forall v \cdot A}{[t / v] A}$

In unserem Rahmen nimmt sie die Gestalt (13b) an. Dort soll $a$ irgendeine Sorte und $v$ eine Variable dieser Sorte sein. Ferner steht $[t / v] A$ für diejenige Formel, die aus $A$ dadurch hervorgeht, dass man dort alle freien Vorkommnisse der Variablen $v$ durch den Term $t$ ersetzt. Dabei gilt die Einschränkung: Der Term $t$ enthält keine Variable $w$, sodass $v$ in $A$ innerhalb einer Teilformel der Gestalt $Q v \cdot B(Q \in\{\exists, \forall\})$ frei vorkommt.

Mit der Formulierung der Regel (UI) befinden wir uns bereits bei Angabe des deduktiven Apparats - der Axiome und Schlussregeln - von PC'. PC' enthält zwei verschiedene Gruppen von Axiomen: „lexikalische Axiome“ (lexical axioms) geben notwendige und hinreichende Bedingungen dafür an, dass ein semantischer Wert zu einem lexikalischen Grundausdruck der Objektsprache gehört. „Phrasale Axiome“ (phrasal axioms) beschreiben, wie die semantischen Werte komplexer Ausdrücke von denen ihrer Komponenten abhängen. Schließlich sollen „Produktionsregeln“ (production rules) sicher stellen, dass zu jedem Satz der Objektsprache auch hinreichende und notwendige Wahrheitsbedingungen ableitbar sind. - Wir geben zunächst die lexikalischen Axiome von PC' an:

14) (LA1) $\operatorname{Val}(\mathbf{t}$, Phil ponders $) \leftrightarrow$ Phil überlegt

(LA2) $\quad$ Val(t, Chris agrees $) \leftrightarrow$ Chris stimmt zu

(LA3) $\quad \mathrm{Val}(\mathbf{t}$, Jill knows Kate $) \leftrightarrow$ Jill kennt Kate

(LA4) $\forall x^{\text {wert }} y^{\text {wert }} \cdot[\operatorname{Val}(\langle x, y\rangle$, and $) \leftrightarrow x=\mathbf{t} \wedge y=\mathbf{t}]$

(LA5) $\forall x^{\text {wert }} y^{\text {wert }} \cdot[\operatorname{Val}(\langle x, y\rangle$, or $) \leftrightarrow x=\mathbf{t} \vee y=\mathbf{t}]$

(LA6) $\forall x^{\text {wert }}$. [Val $(x$, it is not the case that $\left.) \leftrightarrow \neg x=\mathbf{t}\right]$

Nun zu den phrasalen Axiomen! Es ist schon darauf hingewiesen worden dass die in Larson und Segals Axiomen frei vorkommenden Variablen als implizit allquantifiziert gedeutet werden müssen. In der folgenden Liste machen wir diesen stets hinzuzudenkenden initialen Allquantor wieder explizit. Wie oben erklärt, vermeiden wir dabei auch den Gebrauch von Konstanten einer speziellen Sorte als Variablen einer anderen Sorte. 


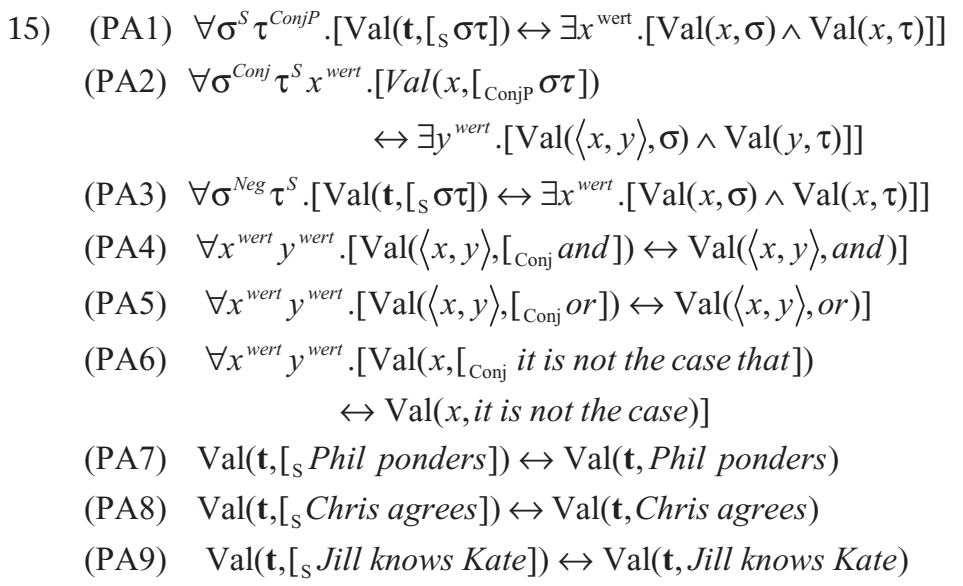

Die Ableitungsregeln oder, wie Larson und Segal sagen, die Produktionsregeln von PC' sind neben der bereits oben (s. (13b)) angeführten Regel (UI) der Allbeseitigung noch die folgenden Schlussweisen:

$$
\begin{aligned}
& \text { 16) (SE) } \frac{A[B] B \leftrightarrow C}{A[C]} \\
& \text { (CJ) } \frac{A \leftrightarrow \exists x y \cdot\left[\operatorname{Val}\left(x, \sigma^{S}\right) \wedge \operatorname{Val}\left(y, \tau^{S}\right) \wedge x=\mathbf{t} \wedge y=\mathbf{t}\right]}{A \leftrightarrow \operatorname{Val}\left(\mathbf{t}, \sigma^{S}\right) \wedge \operatorname{Val}\left(\mathbf{t}, \tau^{S}\right)} \\
& \text { (DJ) } \frac{A \leftrightarrow \exists x y \cdot\left[\operatorname{Val}\left(x, \sigma^{S}\right) \wedge \operatorname{Val}\left(y, \tau^{S}\right) \wedge[x=\mathbf{t} \vee y=\mathbf{t}]\right]}{A \leftrightarrow \operatorname{Val}\left(\mathbf{t}, \sigma^{S}\right) \vee \operatorname{Val}\left(\mathbf{t}, \tau^{S}\right)} \\
& \text { (Neg) } \frac{A \leftrightarrow \exists x^{\text {wert }} \cdot\left[\neg x=\mathbf{t} \wedge \operatorname{Val}\left(x, \sigma^{S}\right)\right]}{A \leftrightarrow \neg \operatorname{Val}\left(\mathbf{t}, \sigma^{S}\right)}
\end{aligned}
$$

Die Regel (SE) („substitution of equivalents“) ist dabei so zu verstehen, dass $A[C]$ dadurch aus $A[B]$ hervorgeht, dass man in $A[B]$ die Teilformel $B$ an einigen Stellen ihres Vorkommens durch $C$ ersetzt. - Damit haben wir nun - in leicht veränderter Form - alle Bestimmungsstücke der Theorie PC' beisammen, die Larson und Segal selbst angeben. Wie wir im nächsten Unterabschnitt gleich sehen werden, fehlen aber noch einige Regeln, damit die Theorie so arbeitet, wie es sich ihre Urheber wünschen. 
Davon abgesehen benötigen wir auch noch eine Reihe von Axiomen, um die Regel (UI) überhaupt anwenden zu können. In Larsons und Segals ursprünglicher Formulierung sieht etwa das Axiom (PA1) folgendermaßen aus:

17) $\operatorname{Val}\left(\mathbf{t},\left[{ }_{\mathrm{S}} \mathrm{S}\right.\right.$ ConjP] $\left.]\right)$ iff for some $\mathrm{z} \operatorname{Val}(z, \mathrm{~S})$ and $\operatorname{Val}(\mathrm{z}, \mathrm{ConjP})$.

Um hier das zweite und dritte Vorkommnis von S durch den Term [S Phil ponders] ersetzen zu können, müssen wir wissen, dass dieser Term von der Sorte $S$ ist. Dieses Wissen steht uns aber in Larsons und Segals Version von PC' gar nicht zur Verfügung. Streng genommen sind alle Anwendungen, die sie von der Regel (UI) machen, fehlerhaft.

Nun wird man einwenden, dass es ja offensichtlich ist, dass der Term [ Phil ponders] zur Sorte $S$ gehört, da in der Grammatik (1) die Kette Phil ponders aus dem Symbol S ableitbar ist. Aufgabe einer Formalisierung ist es aber u. a., solche offensichtlichen Trivialitäten zu kontrollieren und so z. B. sicherzustellen, dass man nichts - auch nichts Triviales - vergessen hat. In PC' muss in irgendeiner Weise klar gemacht werden, zu welchen Sorten die einzelnen strukturellen Bezeichnungen der in (1) ableitbaren Ketten gehören. Letztendlich geht es darum, wie syntaktische Informationen für PC' bereitgestellt werden können. Das von Tarski (1935) angegebene Beispiel einer Wahrheitstheorie für die Sprache der Klassenlogik enthält eine komplette axiomatisierte Syntax für diese Sprache; vgl. Tarski (1935: 279-295). Eine solche Lösung kommt aber für PC' aufgrund der Einbettung dieser Theorie in eine modulare Architektonik nicht in Frage. Das semantische Modul sollte nicht eine Kopie des syntaktischen Moduls enthalten, vielmehr sollte Letzteres dem Ersteren die erforderlichen syntaktischen Informationen liefern. Hier fehlt also noch ein „Interaktionsprinzip“ für die Zusammenarbeit der beiden Module.

Wir haben in unserer Formulierung der Regel (UI) durch die erste Prämisse dieser Regel gefordert, dass der einzusetzende Term zur Sorte der zu ersetzenden Variable passt. Wir haben aber bisher keine Möglichkeit, irgendeine dieser Zusatzprämissen zu beweisen. Da im Semantikmodul nichts Syntaktisches bewiesen werden soll, ist dies aber auch durchaus richtig so. Das Syntaxmodul muss der Semantik die relevanten Informationen zuliefern. Wir formulieren dazu das folgende Interaktionsprinzip, welches der Theorie PC' noch unendlich viele Axiome hinzufügt.

Ist $t$ Strukturterm einer korrekten Ableitung in der Grammatik (1), die mit dem nonterminalen Symbol $E$ beginnt, so ist $\mathrm{D}^{E}(t)$ ein Axiom von PC'.

Die nach (18) gewonnenen Axiome wollen wir „syntaktische Axiome“ nennen. - Wir wollen nun ein Anwendungsbeispiel der Theorie PC' etwas genauer anschauen. 


\subsection{Anwendungsbeispiel}

Hierbei geht es um den (hier bereits in seiner analysierten Form angegebenen) Satz:

19) $\left[{ }_{S}[\right.$ Phil ponders $]\left[{ }_{\text {Conjp }}\left[{ }_{\text {Conj }}\right.\right.$ or $]\left[{ }_{\mathrm{S}}\right.$ Chris agrees $\left.\left.]\right]\right]$;

vgl. Larson und Segal (1995: 86f). Um die folgenden Formeln etwas übersichtlicher zu gestalten, kürzen wir diese Struktur durch ihre Nummer (19) ab. Das syntaktische Modul liefert uns die Informationen

20) (a) $\mathrm{D}^{S}\left(\left[_{\mathrm{S}}\right.\right.$ Phil ponders $\left.]\right)$ und

(b) $\mathrm{D}^{\text {Conjp }}\left(\left[_{\text {Conjp }}\left[\left[_{\text {Conj }}\right.\right.\right.\right.$ or $][$ S Chris agrees $\left.\left.]\right]\right)$.

Zusammen mit dem einschlägigen phrasalen (PA1) führt zweimalige Anwendung von (UI) auf

21) $\operatorname{Val}(\mathbf{t},(19)) \leftrightarrow \exists x^{\text {wert }} \cdot[\operatorname{Val}(x,[$, Phil ponders $])$

$\wedge \operatorname{Val}\left(x,\left[_{\text {Conjp }}\left[{ }_{\text {Conj }}\right.\right.\right.$ or $][$ S Chris agrees $\left.\left.\left.]\right]\right)\right]$.

Dies ist entspricht der Nr. (39) bei Larson/Segal (1995: 86). Die beiden Autoren analysieren nun zunächst die Konjunktionalphrase mit Hilfe des Axioms (PA2) und gelangen so zu einer Entsprechung von:

22) $\operatorname{Val}\left(x^{\text {wert }},\left[{ }_{\text {Conjp }}\left[{ }_{\text {Conj }}\right.\right.\right.$ or $]\left[{ }_{\mathrm{S}}\right.$ Chris agrees $\left.\left.]\right]\right)$

$\leftrightarrow \exists y^{\text {wert }}\left[\operatorname{Val}\left(\langle x, y\rangle,\left[{ }_{\text {Conj }}\right.\right.\right.$ or $\left.]\right) \wedge \operatorname{Val}(y,[$ [S Chris agrees $\left.])\right]$.

Dieser Schritt ist (mit Hilfe der entsprechenden syntaktischen Axiome ${ }^{11}$ ) auch in unserer Version von PC' vollziehbar. Für das linke Konjunktionsglied im Skopus des Existenzoperators von (22) erhält man mit Hilfe von (PA5), (LA5) und der Regel (SE):

23) $\operatorname{Val}\left(\langle x, y\rangle,\left[{ }_{C o n j}\right.\right.$ or $\left.]\right) \leftrightarrow x=\mathbf{t} \vee y=\mathbf{t}$.

Anwendung von (SE) auf (22) und (23) liefert:

24) $\operatorname{Val}\left(x^{\text {wert }},\left[{ }_{\text {Conip }}\left[{ }_{\text {Conj }}\right.\right.\right.$ or $]\left[{ }_{\mathrm{S}}\right.$ Chris agrees $\left.\left.]\right]\right)$

$\leftrightarrow \exists y^{\text {wert }}\left[[x=\mathbf{t} \vee y=\mathbf{t}] \wedge \operatorname{Val}\left(y,\left[{ }_{\mathrm{S}}\right.\right.\right.$ Chris agrees $\left.\left.]\right)\right]$.

Wenn wir dies nun mittels (SE) in (21) einsetzen, so erhalten wir: 


$$
\begin{aligned}
& \operatorname{Val}(\mathbf{t},(19)) \leftrightarrow \exists x^{\text {wert }} .\left[\operatorname{Val}\left(x,\left[{ }_{\mathrm{s}} \text { Phil ponders }\right]\right)\right. \\
& \left.\wedge \exists y^{\text {wert }}\left[[x=\mathbf{t} \vee y=\mathbf{t}] \wedge \operatorname{Val}\left(y,\left[{ }_{\mathrm{s}} \text { Chris agrees }\right]\right)\right]\right]
\end{aligned}
$$

Hier endet nun aber jeder weitere Ableitungsversuch. Es ist nicht möglich, mit den in PC' vorhandenen Mitteln (25) in einen W-Satz umzuformen. Die Ableitung, die Larson und Segal (1995: 86) angeben, beruht auf einer Erschleichung: sie wenden stillschweigend zwei Regeln an, die gar nicht zu PC' gehört. Von (25) gehen sie nämlich zunächst zu (einer Entsprechung von)

$$
\begin{aligned}
\operatorname{Val}(\mathbf{t},(19)) \leftrightarrow & \exists x^{\text {wert }} y^{\text {wert }} \cdot\left[\operatorname{Val}\left(x,\left[{ }_{\mathrm{S}} \text { Phil ponders }\right]\right)\right. \\
& \wedge[x=\mathbf{t} \vee y=\mathbf{t}] \wedge \operatorname{Val}(y,[\text { S Chris agrees }])]
\end{aligned}
$$

über; das ist ihre Nr. (44b). Dieser Übergang ist zwar logisch korrekt - er beruht auf dem Gesetz

$$
A \wedge \exists x . B[x] \leftrightarrow \exists x \cdot[A \wedge B[x]]
$$

(für ein $A$, in dem $x$ nicht frei vorkommt) - , aber es gibt keine ihm entsprechende Regel in PC'. Auf (26) wenden nun Larson und Segal die Regel (DJ) an, was offensichtlich wiederum nicht möglich ist, das diese Regel verlangt, dass der adjunktive "Gleichungsteil“ auf der rechten Seite ganz zum Schluss steht. Hier werden also

$$
\begin{aligned}
& \text { (a) } A \wedge B \leftrightarrow B \wedge A \\
& \text { (b) } A \wedge[B \wedge C] \leftrightarrow[A \wedge B] \wedge C
\end{aligned}
$$

(Kommutativität und Assoziativität der Konjunktion) oder entsprechende Regeln vorausgesetzt. Mit denen gewinnt man natürlich leicht aus (26)

$$
\begin{aligned}
\operatorname{Val}(\mathbf{t},(19)) \leftrightarrow & \exists x^{\text {wert }} y^{\text {wert }} \cdot\left[\operatorname{Val}\left(x,\left[{ }_{S} \text { Phil ponders }\right]\right)\right. \\
& \left.\wedge \operatorname{Val}\left(y,\left[{ }_{\mathrm{S}} \text { Chris agrees }\right]\right) \wedge[x=\mathbf{t} \vee y=\mathbf{t}]\right]
\end{aligned}
$$

Eine Awendung von (DJ) liefert dann

$$
\begin{aligned}
\operatorname{Val}(\mathbf{t},(19)) \leftrightarrow & \operatorname{Val}\left(\mathbf{t},\left[{ }_{\mathrm{S}} \text { Phil ponders }\right]\right) \\
& \vee \operatorname{Val}\left(\mathbf{t},\left[{ }_{\mathrm{S}} \text { Chris agrees }\right]\right),
\end{aligned}
$$

woraus man mit der Regel (SE) und den lexikalischen Axiomen (LA1) und (LA2) die gewünschte Formel (31) erschließt. 
Dieser W-Satz ist aber in PC' nicht ableitbar. Damit erweist sich PC' als Wahrheitstheorie unbrauchbar. Um (31) ableitbar zu machen, muß man zu dieser Theorie (zumindest) noch Prinzipien wie das Verschiebegesetz (27) und die Konjunktionsgesetze (28) hinzufügen. Man benötigt also, wie bereits festgestellt wurde, sehr viel mehr Logik als Larson und Segal erwarten - und dies schon bei einer nahezu trivialen Objektsprache!

\section{Das Generelle Problem}

Ich werde nicht versuchen, die Theorie PC' zu verbessern. Es wurde ja schon gesagt, was man noch hinzufügen muss, um alle W-Sätze ableitbar zu machen. Damit ist aber erst ein Ziel erreicht: die Ableitbarkeit aller W-Sätze. Das andere Ziel, dass nämlich hierzu der schwächste logische Apparat verwendet werden soll, ist nicht so leicht zu erreichen. Wir wollen nun überlegen, was dieses Ziel eigentlich bedeutet.

\subsection{Logiken - ganz generell}

Der Kernbegriff, um dessen Erfassung es der Logik in erster Linie geht, ist die logische Folgebeziehung. Auf einer sehr abstrakten Ebene kann man eine Logik direkt mit einer solchen Folgebeziehung identifizieren. Eine Folgebeziehung ist eine Beziehung zwischen Satzmengen (Prämissenmengen) und einzelnen Sätzen einer fest vorgegebenen Sprache. Wir schreiben „M Folg $A$ ", wenn die Formel $A$ im Sinne der Beziehung Folg aus der Formelmenge $M$ folgt. Wir nennen eine Formel $A$ eine „Tautologie“ (der Folgebeziehung Folg), wenn $A$ aus der leeren Menge von Voraussetzungen folgt, wenn also $\varnothing$ Folg $A$ gilt. Taut(Folg) soll die Menge der Tautologien der Folgebeziehung Folg sein.

Welche Beziehungen man als Folgebeziehungen ansieht, legt man durch Axiome fest. Ein denkbares und vielfach verwendetes Axiomensystem dieser Art ist etwa:

(A1) $M$ Folg $A$ für jedes $A \in M$.

(A2) Falls $M$ Folg $A$, so auch $N$ Folg $A$ für jedes $N \supseteq M$.

(A3) Falls $M$ Folg $B$ und $\{B\} \cup N$ Folg $A$, so auch $M \cup N$ Folg $A$.

Alle Relationen Folg, die diese Axiome erfüllen, werden als Folgerungsbeziehungen angesehen. Weitere Zusatzaxiome sind denkbar, die zu spezielleren Folgebegriffen führen. Man könnte etwa verlangen, dass alles was aus einer Prämissenmenge $M$ folgt, sich bereits aus einer endlichen Teilmenge von $M$ ergibt.

Mit Hilfe eines Axiomensystems, d. h. einer Menge $A x$ von Axiomen und einer Menge Reg von Regeln kann man eine Folgerelation Folg festlegen, indem man bestimmt, dass $M$ Folg $A$ genau dann gelten soll, wenn sich $A$ mit Hilfe der Regeln aus Reg aus den Annahmen von $M$ und den Axiomen von $A x$ ergibt. Offensichtlich sind dann die Theoreme des Axiomensystems genau die Tautologien der durch das System festgelegten Folgebeziehung. Für genauere Details sei hier auf die Monographie Wójcicki (1988: Kap. 2) verwiesen. So haben wir z. B. gerade mit der axiomatischen Theorie PC' eine Folgebeziehung festgelegt. 
Wichtig für uns ist noch, dass die verschiedenen Folgebeziehungen (über einer Sprache) nicht unverbunden nebeneinander stehen, sondern ein System mit einer wohlbekannten Struktur bilden. Man sieht z.B. leicht, dass der Schnitt Folg $\cap$ Folg zweier Folgebeziehung (über derselben Sprache) wiederum eine Folgebeziehung ist, die in den beiden Ausgangsbeziehungen (mengentheoretisch) enthalten ist. Man kann auch eine Vereinigungsoperation für Folgebeziehungen definieren, die aber nicht einfach mit der mengentheoretischen $\cup$-Operation zusammenfällt. Die Vereinigung zweier Folgebeziehungen enthält aber die beiden miteinander vereinigten Beziehungen wieder als (mengentheoretische) Teile.

Insgesamt erweist sich die Gesamtheit aller Folgebeziehungen als durch die Teilmengenbeziehung geordnet. Dabei handelt es sich um einen speziellen Ordnungstyp, den man als „Verbandsordnung“ bezeichnet. Man sagt, die Folgebeziehungen bilden einen Verband, in dem der Schnitt und die gerade angesprochene Vereinigung die Verbandsoperationen sind und die Teilmengenbeziehung die Verbandsordnung ist; vgl. etwa die Monographie Wójcicki (1988: 36-43).

\subsection{Das Minimalitätsproblem}

Wir sind nun in der Lage, das von Larson und Segal aufgeworfene Problem, etwas genauer formulieren zu können. Wir nennen es das „Minimalitätsproblem“.

M i n i malitätsproble m: Gegeben sei eine interpretierte Objektsprache $S$ zusammen mit einer Metasprache $L$, die hinreichend ausdrucksstark ist, um in ihr W-Sätze für $S$ formulieren zu können. WS $(S)$ sei die Menge aller korrekten, in $L$ formulierten WSätze von $L$. Das Problem besteht darin, eine im Verband aller Folgebeziehungen über $L$ minimale Beziehung Folg zu finden, für die 1.) WS $(S) \subseteq \operatorname{Taut}(F o l g)$ und die (2.) den davidsonschen Randbedingungen genügt.

Mit der Forderung (2.) meinen wir, dass die Folgebeziehung durch ein endliches Axiomensystem bestimmbar sein muss usw. (vgl. oben den Abschnitt 2.1). Im dritten Abschnitt hatten wir gesehen, dass die durch PC' festgelegte Folgebeziehung nicht der ersten Forderung der beiden im Minimalitätsproblem genannten Forderungen genügt.

Das Minimalitätsprinzip ist offensichtlich ein interessantes Problem, das auch ganz unabhängig von seiner Motivation durch Larsons und Segals Version des DavidsonProgramms Aufmerksamkeit beanspruchen darf. Es geht ja letztlich darum, wie viel Logik wir in die semantische Metatheorie einer Sprache hineinstecken müssen, damit diese Theorie adäquat wird.

Beim Minimalitätsproblem handelt es sich keineswegs um ein triviales Problem. Es ist eine durchaus gängige Erscheinung, dass verschiedene Folgebeziehungen in ihren Tautologiemengen übereinstimmen. Man kann also durchaus Taut $(F o l g)=\operatorname{Taut}(F o l g ')$ bei Folg $\neq$ Folg' haben. Hiz (1958) konstruiert z.B. eine von der klassischen abweichende Folgerelation, die genau dieselben Tautologien hat wie diese. Es ist also kein vernünftiger Grund dafür ersichtlich, dass die Menge der korrekten W-Sätze die Folgebeziehung der semantischen Metatheorie eindeutig festlegt. 
Vielleicht wird man vermuten, dass dies gerade die der Objektsprache inhärente Logik tut. Dies ist aber keineswegs der Fall, wie folgende Überlegung zeigt. Nimmt man für jeden Satzbuchstaben $p$ eines Systems der klassischen Logik eine metasprachliche Übersetzung $p^{\prime}$ an, sodass der W-Satz $\mathrm{W}(p) \leftrightarrow p^{\prime}$ gilt, so kann man bereits, wenn die üblichen Klauseln der Tarskischen Wahrheitsdefinition zur Verfügung stehen, mit der sogenannten „minimalen Logik“ adäquate W-Sätze für alle komplexen Formeln herleiten. Z.B. hat man $\mathrm{W}(\neg \neg p) \leftrightarrow \neg \neg p^{\prime}$ (aber selbstverständlich nicht $\left.\mathrm{W}(\neg \neg p) \leftrightarrow p^{\prime}\right)$ ). Die minimale Logik ist aber schwächer als die intuitionistische und somit a fortiori auch als die klassische; sie liegt im Folgerungsverband unter diesen Logiken! Für eine wahrheitstheoretische Semantik der klassichen Logik benötigt man also gar nicht alle klassisch gültigen Tautologien.

All diese Überlegungen zeigen wohl, dass Larson und Segal mit ihrem Ansatz interessante Probleme aufgeworfen haben, obwohl sie bereits bei einem ihrer einfachsten Beispiele den Forderungen ihres eigene Programms nicht nachkommen konnten.

\section{LITERATUR}

Davidson, Donald (1984), Inquiries into truth and interpretation, Oxford: Clarendon Press.

Davies, Martin (1981), Meaning, quantification, necessity, London: Routledge and Kegan.

Glubrecht, Jürgen-Michael, Arnold Oberschelp, Günter Todt (1983), Klassenlogik. Mannheim: Bibliographisches Institut.

Hiz, Henry (1958), „Extendible sentential calculi“, The journal of symbolic logic, 24 (1958), pp. 193 - 202.

Larson, Richard, Gabriel Segal (1995), Knowledge of meaning. An introduction to semantic theory, Cambridge/Mass.: MIT.

Martin, Richard M. (1958), Truth and denotation. A study in semantical theory, Chicago: Chicago University Press.

Tarski, Alfred (1935), „Der Wahrheitsbegriff in den formalisierten Sprachen“, Studia philosophica, I (1935), pp. 261 - 405. - Wieder (mit der Originalpaginierung) in: Berka, Karel, Lothar Kreiser (Hgg.) (1971), Logik-Texte. Kommentierte Auswahl zur Geschichte der Logik, Berlin: Akademie-Verlag, pp. 447 - 559.

Wójcicki, Rzsyard (1988), Theory of logical calculi. Basic theory of consequence operations. Dordrecht: Kluwer.

\section{ANMERKUNGEN}

1 Eine etwas ausführlichere Skizze der wahrheitstheoretischen Semantik geben wir unten im Abschnitt 2.1. Die Originalarbeiten Davidsons, in denen er seine wahrheitstheoretische Semantikkonzeption entwickelt, findet man in dem Sammelband Davidson (1984). Allerdings sind Davidsons Ausführungen eher philosophisch-programmatische Entwürfe. Konkrete semantische Analysen einer Vielzahl unterschiedlicher Konstruktionen im Rahmen des wahrheitstheoretischen Ansatzes findet man in der Monographie von Davies (1981). Jedoch sind die von Davies behandelten Objektsprachen formale Kunstsprachen. Ein umfassendes Lehrbuch, das Davidsons Ansatzes im Bereich der linguistischen Semantik entwickelt, ist Larson und Segal (1995). Das in der vorliegenden Untersuchung als Ausgangspunkt diskutierte 
Problem entstammt dieser Monographie.

2 Wie diese "Festlegung" genau geschieht, wird unten im Abschnitt 2.1. erklärt.

3 Larson und Segal formulieren Prinzipien wie die beiden in (4) mit freien Variablen, geben aber für PC' keine Einsetzungsregel für solche an, sondern lediglich eine Regel der All-Beseitigung, die es erlaubt einen initialen Generalisator fortzulassen und die von ihm gebundene Variable durch einen passenden Term zu ersetzen. Zu einem freie Variablen enthaltenden Prinzip ist also stets eine entsprechende Reihe initialer Generalisatoren hinzuzudenken. Im folgenden schreibe ich dieses Quantorenpräfix immer aus.

4 Das hier von Larson und Segal befolgte Verfahrens findet sich bereits, angewandt auf die Interpretation von Prädikaten, bei Martin (1958), der die Val-Relation als „multiple Denotation“ bezeichnet.

5 Daher haben wir auch kein Quantorenpräfix ergänzt, wie es eigentlich in der Fußnote 3 verlangt wird. Ein solches Präfix „ $\forall S^{\prime \prime}$ wäre ja so zu interpretieren, dass zwar das zweite und dritte, nicht aber das erste Vorkommnis der Variablen „S“ gebunden wäre.

6 Die von Larson und Segal (1995: 112) angegebene Formulierung ist mit mehreren Mängeln behaftet. So ist sie z.B. mit Hilfe der Variablen „S“ formuliert, die sonst nur Satzbäumen vorbehalten ist. Die Regel wird aber generell in allen Sorten angewendet.

7 Ich weiche hier beim Aufbau der Theorie PC' in einer ganzen Reihe von Punkten von der Darstellung in Larson und Segal (1995: 79-88, 111f.) ab, indem ich einige Probleme stillschweigend beseitige. Das Hauptproblem, das ich an meiner Version von PC' aufzeige, dass nämlich einige logische Regeln fehlen und damit diese Theorie das von ihr geforderte nicht leisten kann -, gilt auch, sogar in noch höherem Maße für Larsons und Segals Originalversion.

8 Zur mehrsortigen Prädikatenlogik vgl. man etwa die Monographie von Glubrecht, Oberschelp und Todt (1983). Dort werden auch logische Systeme mit Sortenhierarchien behandelt. In solchen Systemen kann eine Sorte Untersorten haben.

9 Chris agrees ist der Baum, der nur aus einem einzigen Knoten besteht, der gerade mit der Wortfolge Chris agrees etikettiert ist.

10 Da wir die Sorten von den syntaktischen Kategorien abgekoppelt haben, erhalten wir einige der „unsinnigen“ Ausdrücke zurück, die man durch die Zulassung mehrere Sorten eigentlich eliminieren will. Diese Ausdrücke sind jedoch nicht weiter störend.

11 Eine Hinzuziehung dieser Axiome erwähnen wir im Folgenden nicht mehr. 